\title{
Commentary on "Theory-Led Design of Instruments and Representations in Learning Analytics: Developing a Novel Tool for Orchestration of Online Collaborative Learning"
}

\author{
Chris Teplovs \\ Digital Innovation Greenhouse, University of Michigan, USA \\ cteplovs@umich.edu
}

\begin{abstract}
This commentary reflects on the contributions to learning analytics and theory by a paper that describes how multiple theoretical frameworks were woven together to inform the creation of a new, automated discourse analysis tool. The commentary highlights the contributions of the original paper, provides some alternative approaches, and touches on issues of sustainability and scalability of learning analytics innovations.
\end{abstract}

Keywords: Learning analytics, scalability, theoretical perspectives, innovation

Kelly, Thompson, and Yeoman (2015, this issue) present a new, automated discourse analysis technique designed to assist with the orchestration of collaborative learning situations and informed by theories from the fields of learning analytics and CSCL. The automated discourse analysis technique they present is provided as an example of a theory-based development process. The authors are to be commended on their valuable contribution to the literature on learning analytics and theory.

As the authors imply, there are advantages that accrue to the development of analytic tools from a strong theoretical base rather than the more common but less powerful atheoretical approach to such development. Off-the-shelf approaches, as they point out, are burdened with their own theoretical biases. The authors' approach of theory-led design, in which theory is used to establish first principles that then inform the development of tools, focuses on establishing function, behaviour, and structure, and then iterating through design improvements. This is an entirely reasonable and coherent approach. The authors identify two main frameworks that they use to inform their work: IRAC (Jones, Beer, \& Clark, 2013) and the "general framework for learning analytics" proposed by Greller and Drachsler (2012). These frameworks are useful for helping us to understand the development of learning analytics tools. In particular, they help us to understand how learning analytics tools are developed. They do not, however, do as good a job at informing us about why learning analytics tools are developed. The question of "why" is framed in terms of theories of group cognition (Stahl, 2006) and orchestration (Dillenbourg \& Jermann, 2010) that come from the field of CSCL. Overall, the authors have successfully woven together two broad theories about how learning analytics tools are developed with two broad theories that explain why particular learning analytics tools are developed. The resulting theoretical space is used to frame the development of their automated discourse analysis tool.

An alternative approach to using theory-led, principle-based design that weaves together multiple 
(2015). Commentary on "Theory-Led Design of Instruments and Representations in Learning Analytics: Developing a Novel Tool for Orchestration of Online Collaborative Learning". Journal of Learning Analytics, 2(2), 44-46. http://dx.doi.org/10.18608/jla.2015.22.4

theoretical frameworks is to frame the work in terms of a single paradigm. My own involvement for 14 years with the Institute for Knowledge Innovation and Technology provided deep enculturation in principle-based tool development. Starting with a series of deep principles about the process of knowledge building (Scardamalia, 2002), we developed software that represented technological supports for those principles. An off-the-shelf approach would have been considerably less expensive but would have introduced technological biases that run contrary to the unpinning theoretical principles. Over many years, the interplay between the technology (Knowledge Forum) and the theory of knowledge building has resulted in improvements to both. The knowledge building communities that have been engaged have also demonstrated impressive gains.

It would have been nice to contemplate how the exploration of the tool and technique presented in the paper could have been used to inform improvement of the theories used to underpin the development of those tools. For example, are there potential improvements or enhancements to the IRAC framework as a result of the researchers' experience with the development of their tool? How does the use of their tool make us rethink group cognition? This feedback is a hallmark of design-based research and represents an extremely challenging framework from which to operate. However, it also represents a clear route forward for the development and improvement of theory in the field of learning analytics, which is keenly needed. The field of learning analytics overall would be strengthened by having a suite of evolving theories that inform design and that are in turn tested and improved through empirical testing of predictions stemming from those theories.

One question that emerges has to do with the sustainability of the development of theory- and principle-based tools and techniques. Are there better models for getting to scale with educational innovations? Those of us at the University of Michigan's new Digital Innovation Greenhouse think there are. Researchers are good at innovating but their creations can seldom be scaled up for widespread adoption. They are focused on creating and testing innovations but typically have little experience with developing software that can be supported as infrastructure. On the other end of the spectrum, Information Technology Services (ITS) organizations are very good at staging and supporting mature software systems. Their skills are ill-matched to the loose, rapid, duct-tape development methods of researchers. It is not possible to take code from a research group and hand it off to ITS for staging at scale. We have tried for several years; the mismatch is too large. This chasm between innovation and infrastructure is present in all kinds of technology transfer. Our team posited that higher education needs a greenhouse for propagation; an interim space that understands both why innovations arrive so fragile and how to make them stronger before they're taken "outdoors." In the world of entrepreneurial business, these spaces are often called "incubators." We have adopted an "incubator" model that seeks to take educational technology and learning analytics innovations and grow them to scale. Time will tell how successful we are. Innovators such as Kelly, Thompson, and Yeoman can help usher in a new era of learning analytics tools and techniques that incorporates not only powerful design frameworks but also concern themselves with the design, development, and deployment of robust and scalable tools and techniques. 


\section{REFERENCES}

Dillenbourg, P., \& Jermann, P. (2010). Technology for classroom orchestration. New Science of Learning: Cognition, Computers and Collaboration in Education (pp. 525-552). New York: Springer. http://dx.doi.org/10.1007/978-1-4419-5716-0_26

Greller, W., \& Drachsler, H. (2012). Translating learning into numbers: A generic framework for learning analytics. Educational Technology \& Society, 15(3), 42-57. Retrieved from http://hdl.handle.net/1820/4506

Jones, D., Beer, C., \& Clark, D. (2013). The IRAC framework: Locating the performance zone for learning analytics. Proceedings of the 30th Australasian Society for Computers in Learning in Tertiary Education Conference (ASCILITE 2013): Electric Dreams, 1-4 December 2013, Sydney, Australia (pp. 446-450). Retrieved from http://eprints.usq.edu.au/24352/

Kelly, N., Thompson, K., \& Yeoman, P. (2015, this issue). Theory-led design of instruments and representations in learning analytics: Developing a novel tool for orchestration of online collaborative learning. Journal of Learning Analytics, 2(2), 14-43. http://dx.doi.org/10.18608/jla.2015.22.3

Scardamalia, M. (2002). Collective cognitive responsibility for the advancement of knowledge. In B. Smith (Ed.), Liberal education in a knowledge society (pp. 67-98). Chicago: Open Court.

Stahl, G. (2006). Group cognition: Computer support for building collaborative knowledge. Cambridge, MA: MIT Press. 